\title{
Excess Low-Frequency Noise in PtSi on p-Type Si Schottky Diodes
}

\author{
Jonathan M. Mooney
}

\begin{abstract}
The power spectrum for platinum silicide on p-type silicon Schottky diodes has been measured for the diodes available on an infrared focal plane array. A careful experimental technique is used to separate the mutual drift of the array as a whole from the drift of the individual diodes. The power spectrum of the noise associated with the diodes appears to be white even for frequencies below $3.0 \times 10^{-5} \mathrm{~Hz}$. This result is compared with recent models of $1 / f$ noise.
\end{abstract}

\section{INTRODUCTION}

$\mathrm{A}$ RRAYS of Schottky diodes consisting of PtSi on p-type Si are widely used as highly sensitive focal plane arrays [1]. Due to local process variations (variations in substrate doping, photolithography variations, or thermal gradients across the active area during diode formation), no two diodes on the focal plane array are identical. Variations in diode photoresponse across the focal plane array lead to nonuniformities in the array output which must be corrected in order to achieve optimal performance. Correction of the nonuniformities is most often achieved by measuring the response of the array to uniform illumination and then using this stored information to correct the relative response variations on a pixel-by-pixel basis [2], [3].

Such correction techniques are effective to the extent that the response of the individual diodes does not change in time. If drift is present between diodes, the coefficients used for the correction must be updated as the drift becomes significant. In order to quantify the performance limit of the correction as a function of time or, conversely, the length of time a correction remains valid, we measured the excess low-frequency noise of the individual diodes on a focal plane array.

The measured diode $1 / f$ noise was surprisingly low; a result that is possibly of interest to a larger community than the users of infrared cameras. This paper is written without explicitly addressing the function of the infrared focal plane arrays, the effect of diode $1 / f$ noise on infrared camera performance, or the impact of these measurements on future infrared cameras. The focus is on the noise measurements and any reference to infrared cameras is included only to help the reader follow the reasoning and illustrate what factors were guiding our intuition.

\section{Experimental Technique Used to Isolate Diode $1 / f$ NOISE}

The majority of the data in the literature on excess low-frequency noise was taken on discreet devices. In this work we used many diodes on a single PtSi focal plane array to obtain

Manuscript received April 13, 1990; revised July 2, 1990. This work was funded by Dr. Horst Wittman at AFOSR under Task 2305J1. The review of this paper was arranged by Associate Editor M. D. Feuer.

The author is with Rome Air Development Center, Hanscom Air Force Base, MA 01731.

IEEE Log Number 9040196. the data. The measurements were made on an infrared camera developed in-house (Rome Air Development Center, Hanscom Air Force Base) that used a David Sarnoff Research Center 160 $\times 244 \mathrm{PtSi}$ infrared focal plane array. The data from each pixel were digitized to $12 \mathrm{~b}$ (0-4095 ADU's). The digital data were transferred to a Hewlett-Packard series 300 computer via the GPIO bus. The camera operated at 30 frames /s. Details on the fabrication of the focal plane array are available in the literature [4].

Fig. 1(a) illustrates a cross section of a unit cell on the focal plane array. Since the Schottky barrier height of the PtSi active area is much less than the bandgap of the Si substrate, the majority of the dark current will arise from emission over the Schottky barrier. The $\mathrm{n}^{-}$guard ring is included to reduce edge effects. The unit cell is operated by biasing the CCD transfer gate to establish the desired surface potential at the Si surface between the $\mathrm{n}^{+}$region and the $\mathrm{n}^{-}$BCCD implant (buried-channel charge-coupled device). The potential of the $\mathrm{PtSi}$ follows the Si surface potential (the two are shorted via the $\mathrm{n}^{+}$region). Electrons flow out of the PtSi into the CCD well until the potential of the PtSi equals that of the Si surface (see Fig. 1(b)). Once the two are in steady state, the positive voltage on the CCD transfer gate is reduced until the PtSi diode is isolated from the CCD multiplexer. The incident photon flux enhances the diode current. The diode current reduces the voltage on the $\mathrm{PtSi}$ as electrons accumulate on the PtSi electrode. The electrons are read out when the surface potential of the $\mathrm{Si}$ is dropped on the following cycle. During a complete frame the voltage across the Schottky diode typically drops from 12 to $9 \mathrm{~V}$.

The response of the camera system was quantified by the mean-variance technique. For a shot-noise-limited process, the variance is equal to the mean when both are measured in number of carriers. When the quantities are measured in units other than number of carriers, the measured units can be referenced to number of carriers by plotting the variance as a function of the mean. The slope of the resulting line gives the conversion factor between the measured units and the number of carriers (e.g., $\mathrm{V} / \mathrm{e}^{-}$) $[3],[5]$. The mean variance plot for a typical pixel of the array is shown in Fig. 2. The linear relationship between mean and variance which is apparent in Fig. 2 indicates shot-noise-limited operation. The slope of the linear relation corresponds to a conversion gain (the number of electrons which correspond to each ADU) of $218 \mathrm{e}^{-} / \mathrm{ADU}$. Ideally, mean variance plots should asymptotically approach a minimum variance for low mean values which reflects the noise floor of the drive electronics. The noise floor is not observed with our camera because the charge transfer efficiency of the CCD was poor at low levels; hence, the array could be operated at levels low enough to observe noise-floor-limited operation. The noise floor for the system is estimated to be 400 electrons/sample. Under normal operating conditions with mean well levels of 4 


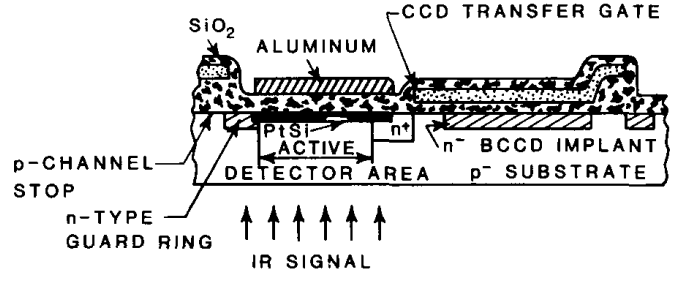

(a)

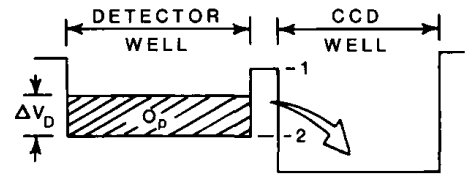

(b)

Fig. 1. Construction and operation of one pixel of the infrared focal plane array. Both the PtSi diode and one phase of the buried channel chargecoupled device readout are shown (taken from [4]).

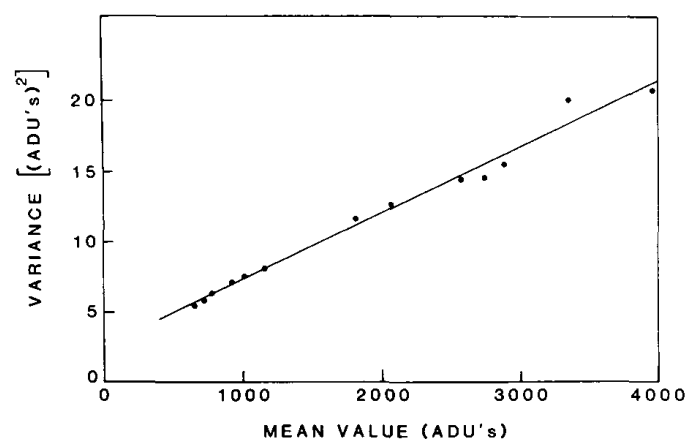

Fig. 2. The variance as a function of the mean for a pixel near the output node on the array. The linearity indicates shot-noise-limited operation.

$\times 10^{5} \mathrm{e}^{-}$, the noise is approximately $1000 \mathrm{e}^{-}$. The noise is larger for pixels away from the output node [3]. Using the value of the conversion gain, we calculated the mean diode current to be $2.0 \times 10^{-12} \mathrm{~A}$, and the current density to be $0.2 \mu \mathrm{A} / \mathrm{cm}^{-2}$.

A photo-generated signal was used to keep the $\mathrm{CCD}$ at a level where the charge-transfer efficiency was optimized. The photogenerated signal was obtained by blocking the entrance pupil of the camera with several sheets of lens tissue. In this state the camera was blind to spatial variations in the surround; however, the camera was still sensitive to changes in the ambient temperature of the room, since the lens tissue itself will heat and cool by convection. The sensitivity of the camera to these thermal variations is approximately $10^{4} \mathrm{e}^{-} / \mathrm{K} /$ pixel. The shot noise from the added photo-generated signal will combine with both the excess noise due to the noise floor and with that due to the poor charge transfer efficiency to mask the $1 / f$ noise

Low-frequency drift in the output node and drive electronics was reduced by using correlated double sampling. Drift due to fluctuations in the transfer voltage and drift in the background temperature will be measured by the camera, and could poten tially be attributed to drift in the diodes. In an effort to eliminate these additional low-frequency noise terms, we used the fact that all of the diodes in one column will see the same transfer voltage and will see approximately the same thermal drifts. We isolated the excess low-frequency noise of the diode itself by using the fact that, if the diodes drift together, the correlated part of the drift is not due to individual diode $1 / f$ noise.

To remove the mutual drift of the diodes, while maintaining reasonable memory and storage requirements, we measured forty pixel values from a single column for each frame. Of the values, 39 were averaged, the average was then subtracted from the value of the fortieth. This process was repeated for $12 \mathrm{~h}$ while saving 1 of every 160 results for each of eight diode groups in different columns on the array. To reduce the variance problem characteristic of Fourier transform routines, we repeated this process eight times. The periodograms for each of the eight runs were calculated and then averaged [6]. We did not use apodization windows since they add confusion to the low-frequency portion of the calculated power spectrum.

The extent to which this approach removes the mutual drift of the diodes is limited by how well the 39-pixel average matches the value of the fortieth pixel. Consider the case where the area of the pixels varies across the array. After the subtraction of the 39-pixel average, the effective response of the resulting signal will be that of the fortieth scaled by the difference between the area of the fortieth and the average value of the area of the 39 pixels. Hence the sensitivity of the resulting signal due to thermal variations of the lens tissue, and mutual drift in the output electronics will be greatly reduced, but it will not be completely removed.

We model the response of the $i$ th pixel as a function of time by

$$
d_{i}(t)=\gamma_{i} g(t)+u_{i}(t)
$$

where $g(t)$ is the part of the diode response that is correlated with the other diodes; it includes the effects of thermal variations in the surround and drift in the bias voltages that drive the array. $\gamma_{i}$ is a factor which determines the pixel's sensitivity to these correlated variations; it includes the scaling effects of the pixel area. $u_{i}(t)$ is the random uncorrelated contribution to the response; it includes the effects of shot noise, diode $1 / f$ noise, clock feed-through noise, and charge-transfer noise. The average of $u_{i}(t)$ over many diodes is assumed to be zero $(\langle u(t)\rangle$ $=0)$. The average of $d_{i}(t)$ over many diodes is

$$
\langle d(t)\rangle=\langle\gamma\rangle g(t) \text {. }
$$

For our measurements, the autocorrelation is calculated in the form of an expectation in the time domain and is given by

$$
\begin{aligned}
R_{d_{i}}(t, t+\tau) & =E\left[d_{i}(t) d_{i}^{*}(t+\tau)\right] \\
& =\gamma_{i}^{2} R_{g}(t, t+\tau)+R_{u_{i}}(t, t+\tau)
\end{aligned}
$$

where $R_{g}(t, t+\tau)$ and $R_{u_{i}}(t, t+\tau)$ are the autocorrelation of $g(t)$ and $u_{i}(t)$, respectively, and we have assumed that $E\left[u_{i}(t) g(t)\right]=0$.

In the procedure used to determine the diode $1 / f$ noise, a spatial average is subtracted from one diode value. From (1) and (2) we define

$$
d_{i}(t)=d_{i}(t)-\langle d(t)\rangle .
$$

The autocorrelation of $\tilde{d}_{i}$ is

$$
R_{d_{i}}(t, t+\tau)=\left(\gamma_{i}-\langle\gamma\rangle\right)^{2} R_{g}(t, t+\tau)+R_{u_{i}}(t, t+\tau) \text {. }
$$

The power spectrum is determined by taking the Fourier transform of (5). If we assume that both $g(t)$ and $u_{i}(t)$ are 


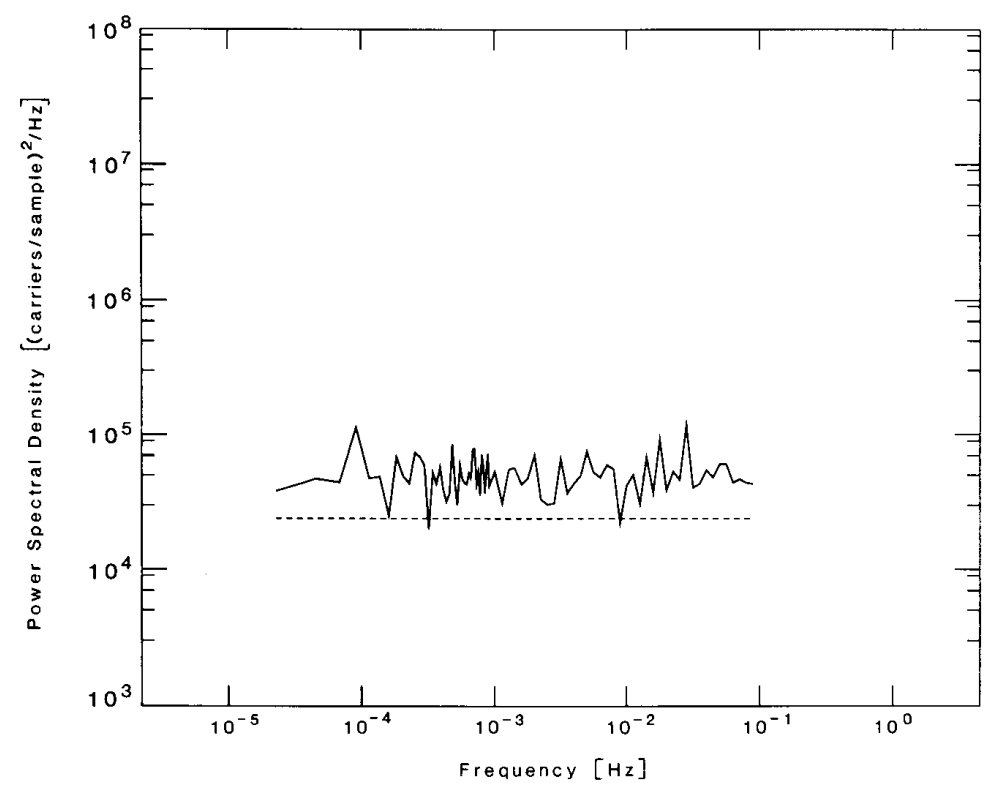

Fig. 3. Estimated power spectrum for a diode with $E[\tilde{d}]=5 \mathrm{ADU}$ 's (solid line). The dashed line is the shot noise limit for the diode.

stationary in the wide sense, the power spectrum is

$$
S_{d_{i}}(f)=\left(\gamma_{i}-\langle\gamma\rangle\right)^{2} S_{g}(f)+S_{u i}(f) .
$$

This equation shows that if sensitivity of the sampled pixel to the variations in the surround is different than that of the multipixel average, then the effect of the surround will be reduced, but not completely removed. The expectation value of $\tilde{d}_{i}(t)$ is

$$
E\left[\tilde{d}_{i}\right]=\left(\gamma_{i}-\langle\gamma\rangle\right) E[g] .
$$

By substituting (7) into (6), we obtain

$$
S_{d_{i}}(f)=\left(E\left[\tilde{d}_{i}\right]\right)^{2} \frac{S_{g}(f)}{(E[g])^{2}}+S_{u_{i}}(f) .
$$

Here the term $S_{g}(f) /(E[g])^{2}$ is the normalized power spectrum of the noise source which affects all of the diodes. Equation (8) shows that if the mutual drift of the diodes is significant, then the power spectrum will vary as the square of $E\left[\tilde{d}_{i}\right]$.

\section{Experimental Results}

Figs. 3-5 exhibit a representative sampling of the observed power spectrum for three of the eight diodes measured. In each of the figures, the solid line connects data obtained by direct calculation of the power spectrum from the measured data as given by (4). Many of the data at the higher frequencies are not shown to reduce the demand on the printer. The dashed line in each figure represents the power spectrum of the shot noise as calculated from the measured mean number of carriers per well.

At first blush, it would appear that some of the diodes exhibit $1 / f$ noise, some do not, while others exhibit excess low-frequency noise which is not quite $1 / f$ in nature. This interpretation is misleading since it is based on the assumption that the procedures used to remove the drift work perfectly. From (8), one can see that the additional contribution to the power spectral density varies as the square of $E\left[\tilde{d}_{i}\right]$. Fig. 6 illustrates this effect in a plot of the estimated value of $S_{d}\left(2.3 \times 10^{-5} \mathrm{~Hz}\right)$ as a function of $E\left[\tilde{d}_{i}\right]$ for all eight diode data sets. Fig. 6 verifies the predictions of $(8)$, which implies that the excess low-frequency contribution to the power spectrum is a result of external influences rather than individual diode drift. Hence of the three figures $(3,4$, and 5$)$, we surmise that only Fig. 3 represents the estimated power spectrum of a $\mathrm{PtSi} / \mathrm{p}$-type Si Schottky diode and that the Schottky diodes on this array are free of $1 / f$ noise to the limit of this measurement. Figs. 4 and 5 are not accurate representations of the diode power spectrum since the effects of drift in room temperature, and drift in the clock and bias levels are not fully removed from the noise measurement.

\section{Comparison of Literature Models to the EXPERIMENTAL DATA}

Currently prevailing theories of Schottky diode $1 / f$ noise suggest that the noise is due to mobility fluctuations in the depletion region of the semiconductor, where the power spectrum of the fluctuations varies as $1 / f$ [7], [8]. To compare our experimental results to models of $1 / f$ noise in Schottky diodes, we must reformulate the models into a form which is valid under reverse bias. The models of Kleinpenning and Luo are similar with the exception of an added integrating factor in the latter. Kleinpenning finds that the power spectrum of the current noise is

$$
S_{K}(f)=\left[\frac{I v_{T}}{\mu W v_{D}}\right]^{2} \int_{x_{m}}^{W} \int_{x_{m}}^{W} S_{\mu}\left(x, x^{\prime}, f\right) d x d x^{\prime}
$$

while Luo finds

$$
\begin{aligned}
S_{L}(f)= & {\left[\frac{I v_{T}}{\mu D}\right]^{2} \int_{x_{m}}^{W} \int_{x_{m}}^{W} S_{\mu}\left(x, x^{\prime}, f\right) } \\
& \cdot e^{-\beta N_{A} W^{2} / \epsilon} e^{\beta\left(\phi(x)+\phi\left(x^{\prime}\right)\right)} d x d x^{\prime}
\end{aligned}
$$




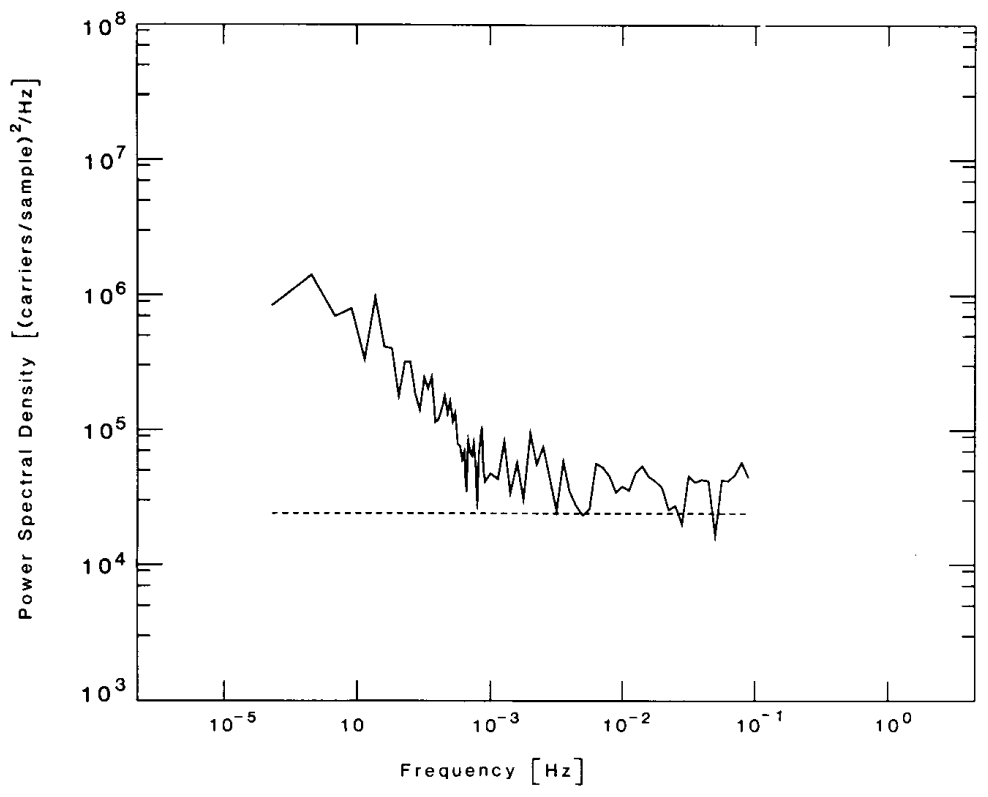

Fig. 4. Similar to Fig. 4 for a diode with $E[\tilde{d}]=54$ ADU's.

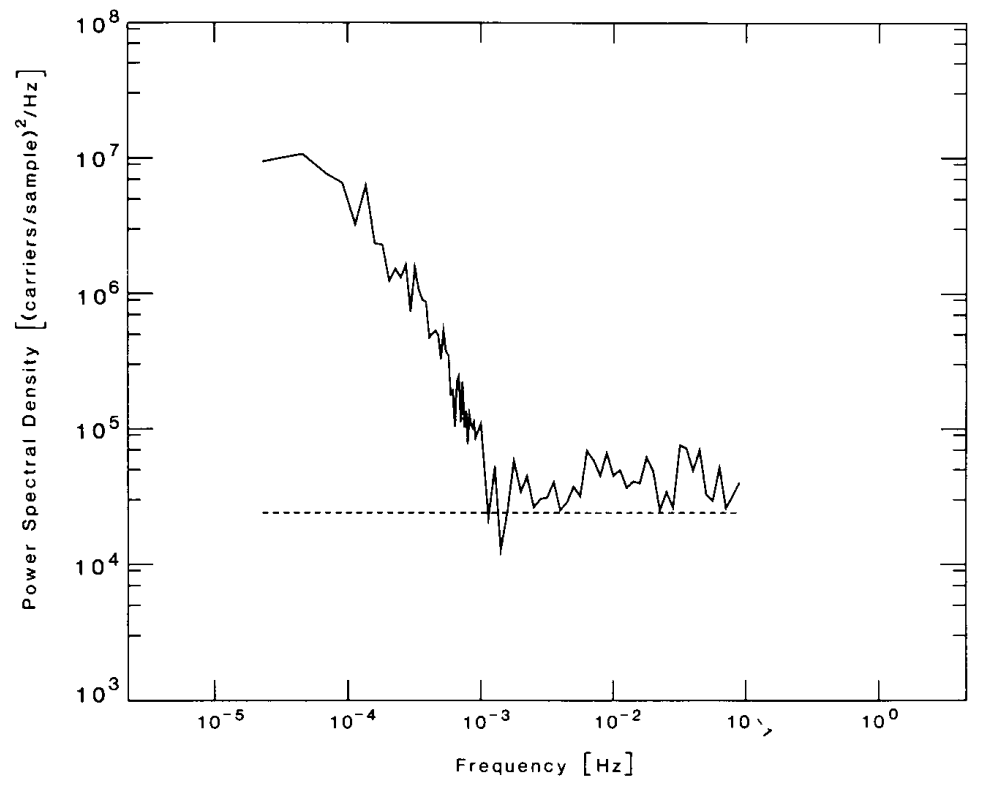

Fig. 5. Similar to Fig. 4 for a diode with $E[d]=143$ ADUs.

where

$$
S_{\mu}\left(x, x^{\prime}, f\right)=\frac{\alpha \mu^{2}}{f p(x) A} \delta\left(x-x^{\prime}\right)
$$

where $q$ is the elemental charge, $\beta=q / k T, A$ is the diode active area, $D$ is the diffusivity, $I$ is the mean current, $\mu$ is the mobility, $\delta\left(x-x^{\prime}\right)$ is the Dirac delta function, $\alpha$ is the Hooge parameter which has a value of between $2 \times 10^{-3}$ and $9 \times$ $10^{-10}$ and is dimensionless [9], [10].
The hole potential in the semiconductor measured relative to the bottom of the valence band in the semiconductor bulk is (following [11] and [12])

$$
\phi(x)=\frac{q N_{A}}{2 \epsilon}(W-x)^{2}-\frac{q}{16 \pi \epsilon x}
$$

where we have included the influence of the Schottky barrier lowering into the hole potential; $N_{A}$ is the dopant concentration; 


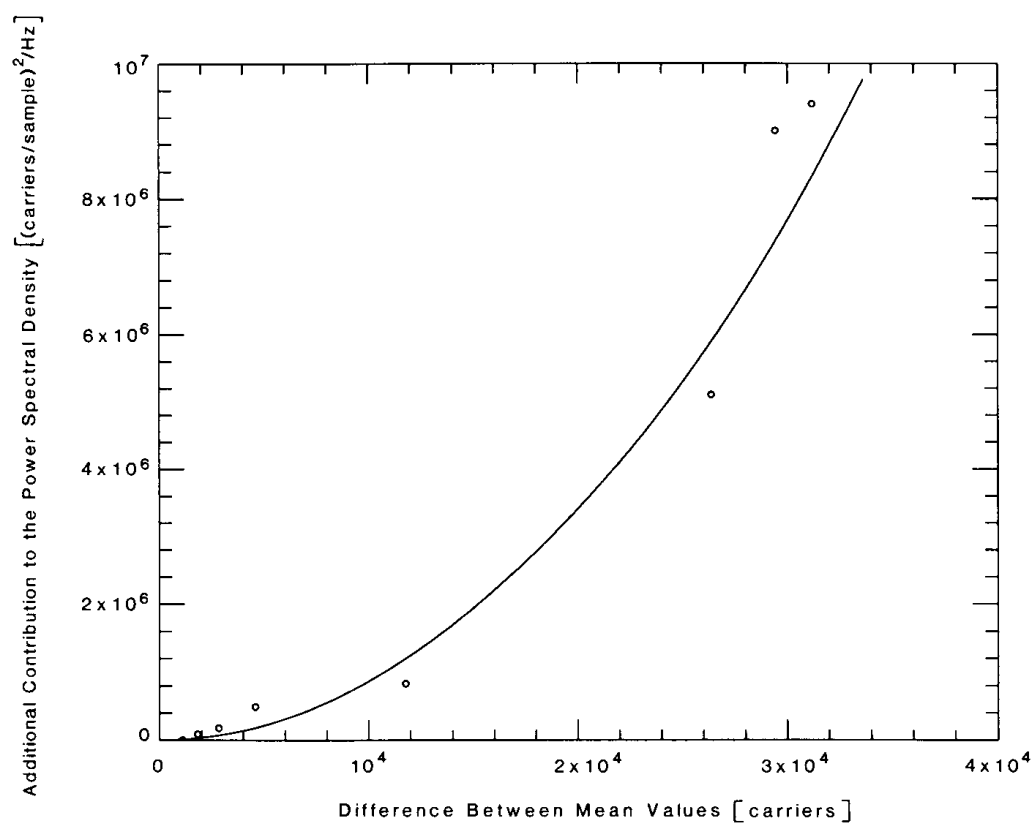

Fig. 6. The estimated value of $S_{N}\left(2.3 \times 10^{-5} \mathrm{~Hz}\right)$ as a function of $E$ [ $d$ ] for each of the eight diodes measured. The solid line represents the results on would expect when (8) is valid. One free parameter $S_{g}\left(2.3 \times 10^{-5} \mathrm{~Hz}\right) /(E[g])^{2}$ was adjusted to obtain the displayed fit.

$\epsilon$ is the permittivity of the semiconductor; $W$ is the depletion region depth

$$
W=\left[\frac{2 \epsilon\left(V_{b i}-V\right)}{q N_{A}}\right]^{1 / 2}
$$

$V_{b i}$ is the built-in voltage of the Schottky contact; and $V$ is the applied forward bias voltage.

The hole concentration as a function of distance from the $\mathrm{PtSi} / \mathrm{Si}$ interface is

$$
p(x)=N_{A} e^{-\beta \phi(x)}+J e^{-\beta \phi(x)} \int_{x}^{W} \frac{e^{\beta \phi(x)}}{\mu k T} d x
$$

where $J$ is the current density, $x_{m}$ is the distance from the $\mathrm{PtSi} / \mathrm{Si}$ interface to the Schottky barrier maximum

$$
x_{m}=\frac{1}{4}\left[\frac{q}{\pi^{2} \epsilon N_{A}\left(V_{b i}-V\right)}\right]^{1 / 4}
$$

$v_{D}$ has the units of a velocity and is given by

$$
\frac{1}{v_{D}}=e^{-\beta \phi\left(x_{m}\right)} \int_{x_{m}}^{W} \frac{q e^{\beta \phi(x)}}{\mu k T} d x
$$

$v_{T}$ is the average velocity of the thermally excited carriers at the Schottky barrier maximum directed normal to the interface

$$
v_{T}=\left[\frac{k T}{2 m^{*} \pi}\right]^{1 / 2}
$$

and $m^{*}$ is the effective mass of valence-band minimum. Since our measurements were made with the diode at $77 \mathrm{~K}$ under reverse bias, the minority carrier contribution to the total current will be more than ten orders of magnitude less than that of the majority carriers and we can neglect minority carrier injection.
This approach is physically different from that of both Kleinpenning and Luo in two ways: 1) the effects of image force lowering are included in the barrier potential profile [the last term in (11)], and 2) the effects of the injected charge are included in hole concentration [the last term in (13)]. Both of these physical effects are more significant for reverse than for forward bias.

Using (9)-(16), one can calculate the expected values of the $1 / f$ power spectrum. To facilitate comparison of the theoretical and experimental results, we calculate the frequency at which the $1 / f$ noise will exceed the shot noise $\left(f_{c}\right)$. By inspection of Fig. 3 , we anticipate that the value of $f_{c}$ will be less than $2 \times$ $10^{-5} \mathrm{~Hz}$. When $|q V| \gg k T$, the power spectrum of the shot noise is

$$
S_{\text {shot }}(f)=2 q I \text {. }
$$

By combining (9)-(17) we can solve for $\left(f_{c}\right)$ in Kleinpenning's model

$$
f_{c k}=\frac{J \alpha}{2 q}\left[\frac{v_{T}}{W v_{D}}\right]^{2} \int_{x_{m}}^{W} \frac{d x}{p(x)}
$$

and in Luo's model

$$
f_{c_{L}}=\frac{J \alpha}{2 q}\left[\frac{v_{T}}{D}\right]^{2} \int_{x_{m}}^{W} \frac{e^{2 \beta \phi(x)}}{p(x)} d x .
$$

Fig. 7 illustrates the dependence of $f_{c}$ on reverse bias where we have used $T=77 \mathrm{~K}, V_{b i}=0.2 \mathrm{~V}, N_{A}=10^{21} 1 / \mathrm{m}^{3}, \mu=0.3460$ $\mathrm{m}^{2} /(\mathrm{V} \cdot \mathrm{s})$. The value of the Hooge parameter was set to the lowest value available in the literature $\left(\alpha=9 \times 10^{-10}\right.$ for Si at $77 \mathrm{~K}$ ) [10] in an effort to obtain the best possible agreement between theory and experiment. From Fig. 7 we can see that in the neighborhood of $-10.0-\mathrm{V}$ bias, Kleinpenning's model gives 


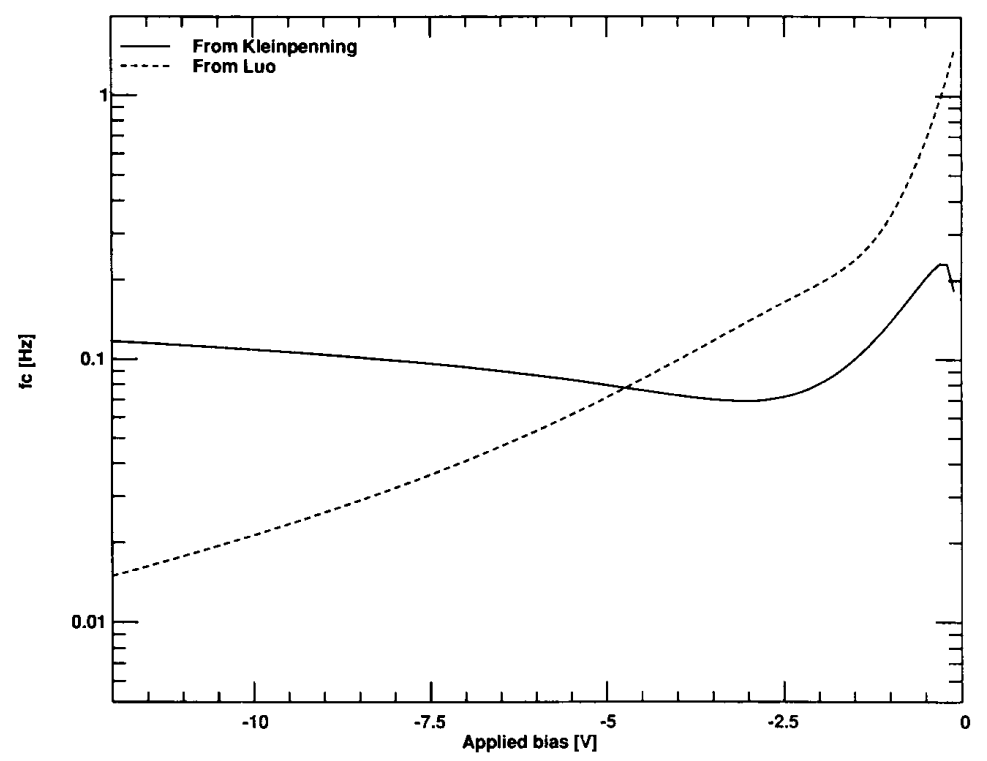

Fig. 7. $f_{c}$ as a function of reverse bias for a PtSi on p-type Si Schottky diode.

a value of approximately $0.1 \mathrm{~Hz}$ for $f_{c}$ while Luo's gives approximately $0.02 \mathrm{~Hz}$, neither of which agrees with the measured result of less than $2 \times 10^{-5} \mathrm{~Hz}$. Further inspection of (18a) and (18b) shows that for photocurrents of the magnitude used during the measurement, no appreciable change in $f_{c}$ is expected despite the fact that the current density appears explicitly in both expressions. This is because $p(x)$ is nearly proportional to $J$ when the diode is under reverse bias (see (13)).

\section{Discussion}

These results are at once surprising and anticipated. The ubiquitous nature of $1 / f$ noise and the fact that Schottky diodes have been shown to exhibit $1 / f$ noise make the result surprising. The fact that nonuniformity correction techniques are successful over many hours leads one to anticipate at most only small amounts of excess low-frequency noise for Schottky diodes. Since Fig. 3 is white down to $2.3 \times 10^{-5} \mathrm{~Hz}$, one concludes that the value of $f_{c}$ is below $2.3 \times 10^{-5} \mathrm{~Hz}$, which is consistent with the observed longevity of nonuniformity corrections, however inconsistent with current $1 / f$ noise models. This inconsistency is troubling and behooves us to reconsider critically our experimental protocols and theoretical development.

A potential flaw in our experiment is in the correlation technique used to remove the mutual drift of the diodes, and in the mean-variance technique used to convert from ADU to $\mathrm{e}^{-}$. Our modeling of the correlation technique has shown that for our specific case, the power spectrum of the white noise is increased by a factor of $(1+1 / 39)$. This deviation should not adversely impact our conclusions. The mean-variance technique is statistical in nature and may give an artificially low value of the conversion gain that will lead to a low estimate of the shot noise. If the white noise floor were artificially high, then it could mask the $1 / f$ noise and reduce the measured value of $f_{c}$. This scenario is unlikely since the performance of the array as an infrared sensor is very close to the theoretical limit, so the noise floor cannot be much greater than the shot noise limit.

Beyond the discrepancy between the $1 / f$ noise models and our experimental results, there is a great deal of experimental data in the literature which typically find larger values of $f_{c}$ than we have measured. For the most part, the power spectrum data for Schottky diodes in the literature exhibit $1 / f$ noise which exceeds that of the shot noise at between 10 and $10^{4} \mathrm{~Hz}$. Noteworthy exceptions are Hsu's data for Pt on n-type Si Schottky diodes at $77 \mathrm{~K}$ [13], and Kleinpenning's data for $\mathrm{Pt} / \mathrm{Ni}$ on ntype $\mathrm{Si}$ at low current levels [8]. In both cases, $1 / f$ noise is observed under certain circumstances, and in neither case is the power spectrum measured below $1 \mathrm{~Hz}$. There are several differences between the diodes and measurement techniques we have used and previous results in the literature. 1) $\mathrm{PtSi}$ on $\mathrm{Si}$ diodes have been shown to be free of interfacial films which are common in many other types of Schottky diodes [14]-[16]. An interfacial film can be a source of interface states which have long lifetimes and may be a source of excess low-frequency noise. 2) Our measurement techniques were unique in that an array of nearly identical diodes was measured simultaneously, and careful attention was paid to the effect that any nonuniformity among the diodes would have on our measurements. No assumption was made about the excess low-frequency noise of the bias supplies, clocks, or amplifier circuits since the effects of these noise sources will be removed by the correlation techniques we implemented. 3) Most other measurements of diode $1 / f$ noise have been obtained with the diodes in forward bias. Our diodes were operated in reverse bias with the dominant current being photocurrent. The output current of diodes under reverse bias will be less sensitive to small bias fluctuations than that of diode under forward bias, which will make the measurement less sensitive to the $1 / f$ noise of the voltage supply. The fact that the dominant current was photocurrent may also play a role in the measurement, but at this time it is unclear how.

\section{Conclusions}

The excess low-frequency noise of PtSi / p-type Si Schottky diodes is at least three orders of magnitude below the predictions of current $1 / f$ noise theories. The noise is also significantly lower than the values commonly reported in the literature for various other kinds of diodes and electronic structures. 
A measurement technique that is capable of reducing the effects of variations in bias voltages and room temperature was shown to be useful for removing correlated ambient low-frequency variations from those of individual diode device noise. Care must be taken when using this technique to insure that the ensemble reference is matched to the diode under test. For PtSi infrared focal plane arrays the technique was found to be useful for measuring the power spectrum down to $10^{-5} \mathrm{~Hz}$.

\section{ACKNOWLEDGMENT}

The author wishes to thank B. Ewing, K. Spaziani, and J. Rex for the design and fabrication of the PtSi camera; J. Mead, J. Murguia, F. Shepherd, J. Silverman, P. Pellegrini, F. Euler, and D. Scribner for helpful discussions; S. DeVore and V. Vickers for supplying the fast Fourier transform routine; and $\mathbf{L}$. Bouthillette for graphical assistance.

\section{REFERENCES}

[1] F. D. Shepherd, "Silicide infrared staring sensors," Proc. SPIE, vol. 930 , p. $1,1988$.

[2] W. S. Ewing, "Silicide mosaic array compensation," in Technical Issues in Infrared Detectors and Arrays, E. Krikorian, Ed., Proc. SPIE, vol, 409, pp. 102-106, 1983.

[3] J. E. Murguia, J. M. Mooney, and W. S. Ewing, "Diagnostics on a PtSi infrared imaging array," in Infrared Technology XIV, I. J. Spiro, Ed., Proc. SPIE, vol. 972, pp. 15-25, 1988.

[4] W. F. Kosonocky, F. V. Shallcross, T. S. Villani, and J. V. Groppe, " $160 \times 244$ element PtSi Schottky-barrier IR-CCD image sensor," IEEE Trans. Electron Devices, vol. ED-32, p. 1564, 1985

[5] L. Mortara and A. M. Fowler, "Evaluations of charge-coupled device (CCD) performance for astronomical use," in Solid State Imagers for Astronomy, J. C. Geary and D. W. Latham, Eds., Proc. SPIE, vol. 290, 1981.

[6] P. D. Welch, "The use of fast Fourier transform for the estimation of power spectra: a method based on time averaging over short, modified periodograms," IEEE Trans. Audio Electroacoust., vol. AU-15, p. 70, 1967.

[7] M. Y. Luo, G. Bosman, A. Van Der Ziel, and L. L. Hench, "Theory and experiments of $1 / f$ noise in Schottky-barrier diodes operating in the thermionic-emission mode," IEEE Trans. Electron Devices, vol. ED-32, pp. 1351-1356, 1985
[8] T. G. M. Kleinpenning, "Low-frequency noise in Schottky barrier diodes," Solid-State Electron., vol. 22, pp. 121-128, 1979.

[9] F. N. Hooge, "Discussion of recent experiments on $1 / f$ noise," Physica, vol. 60 , pp. 130-144, 1972.

[10] G. S. Kousik, C. M. Van Vliet, G. Bosman, and P. H. Handel, "Quantum $1 / f$ noise associated with ionized impurity scattering and electron-phonon scattering in condensed matter," $A d v$. in Physics, vol. 35, pp. 1351-1356, 1988

[11] J. G. Simmons and G. W. Taylor, "Generalized theory of conduction in Schottky barriers," Solid-State Electron., vol. 26, p. 705,1983

[12] S. M. Sze, Physics of Semiconductor Devices. New York, NY: Wiley, 1981, pp. 255-263.

[13] S. T. Hsu, "Low-frequency excess noise in metal-silicon Schottky barrier diodes," IEEE Trans. Electron Devices, vol. ED-17, p. 496, 1970 .

[14] C. A. Crider, J. M. Poate, J. E. Rowe, and T. T. Sheng, "Platinum silicide formation under ultrahigh vacuum and controlled impurity ambients," J. Appl. Phys., vol. 52, p. 2860, 1981.

[15] C. Canali, C. Catellani, M. Prudenziati, W. H. Wadlin, and C. A. Evans, " $\mathrm{Pt}_{2} \mathrm{Si}$ and $\mathrm{PtSi}$ formation with high-purity $\mathrm{Pt}$ thin films," Appl. Phys. Lett., vol. 31, p. 43, 1977.

[16] J. R. Abelson, K. B. Kim, D. E. Mercer, C. R. Helms, R. Sinclair, and T. W. Sigmon, "Disordered intermixing at the platinum: silicon interface demonstrated by high-resolution crosssectional transmission electron microscopy, Auger electron spectroscopy, and MeV ion channeling," J. Appl. Phys., vol. 63, p. $689,1988$.

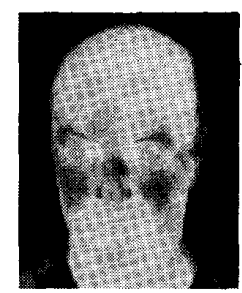

Jonathan M. Mooney was born in Orange, $C A$. He received the $B$. A. degree in physics from the University of California at San Diego in 1981 and the Ph.D. degree in optical sciences from the University of Arizona, Tucson, in 1986 .

He is currently employed in the Electronic Device Technology Division of the Rome Air Development Center, Hanscom AFB, MA. Dr. Mooney is a member of Sigma $\mathrm{Xi}$ and SPIE.

The author's photograph has been taken with an infrared camera using the Schottky-diode focal plane array, the device discussed in the paper. 